\title{
Early Postoperative Unstimulated Serum Thyroglobulin Predicts Utility of Radioactive Iodine in Patients with Papillary Thyroid Cancer
}

\section{Maria Papaleontiou}

Division of Metabolism, Endocrinology and Diabetes; Department of Medicine, University of Michigan School of Medicine, Ann Arbor, Michigan, U.S.A.

Review of: McDow AD, Shumway CM, Pitt SC, Schneider DF, Sippel RS, Long KL 2019 Utility of early postoperative unstimulated thyroglobulin in influencing decision making in patients with papillary thyroid carcinoma. Ann Surg Oncol. Epub 2019 Jul 2. PMID: 31267301.

\section{SUMMARY}

\section{Background}

Papillary thyroid cancer (PTC) is the most common type of differentiated thyroid cancer. Following initial therapy, serum thyroglobulin levels and neck ultrasonography are the mainstay for long-term surveillance to detect persistence or recurrence in these patients (1). Additionally, the most recent guidelines from the American Thyroid Association (ATA) suggest that postoperative thyroglobulin levels may be utilized to guide the use of radioactive iodine (RAI), depending on risk stratification and response to therapy (1).

Even though postoperative levels of thyroglobulin reach a nadir by 3 to 4 weeks, most previous studies have focused on the assessment of response to therapy using thyroid-stimulating hormone (TSH)stimulated thyroglobulin levels obtained 6 to 18 months after initial therapy $(2,3)$. Furthermore, only a few studies have assessed the utility of unstimulated thyroglobulin levels postoperatively, mostly $\geq 3$ months following initial therapy $(1,4)$. The main objective of this study (5) was to evaluate the utility of early postoperative unstimulated thyroglobulin levels (approximately 6 weeks following surgery) as a tool to guide decision making in the use of RAl in patients with PTC.

\section{Methods}

This was a retrospective, single-institution study of patients surgically treated for PTC between 2015 and 2017. Patients with follicular thyroid cancer, with poorly differentiated thyroid cancer, undergoing lobectomy, only and/or with incomplete preoperative or postoperative data were excluded. Patients with thyroglobulin antibodies and those who had not achieved appropriate TSH suppression were also excluded. Appropriate TSH suppression followed the most recent ATA guidelines (1).

Unstimulated thyroglobulin levels were assessed approximately 6 weeks postoperatively, and a level of $\leq 0.2 \mathrm{ng} / \mathrm{ml}$ was used to define excellent response postoperatively. Cervical ultrasounds at 6 and 12 months postoperatively were evaluated to assess for structural evidence of persistent or recurrent disease. Persistent disease was defined as an unstimulated thyroglobulin $>0.2 \mathrm{ng} / \mathrm{ml}$, abnormal cervical ultrasound, or persistent elevation of thyroglobulin antibodies at 6 months after initial therapy (6). Recurrent disease was defined as evidence of disease following previous achievement of an undetectable thyroglobulin level, negative thyroglobulin antibodies, and negative ultrasound (6). Statistical analyses were conducted with primary outcomes 
being evidence of persistent or recurrent disease at 6 months, on the basis of an unstimulated thyroglobulin level and structural disease on ultrasound.

\section{Results}

Of 255 eligible patients, a total of 134 met the inclusion criteria. The mean patient age was 45 years. The majority of patients were female (74.6\%) and Caucasian (92.5\%). Overall, $53.7 \%$ of patients received RAI postoperatively.

A total of $49.3 \%$ of patients achieved an unstimulated thyroglobulin level of $\leq 0.2 \mathrm{ng} / \mathrm{ml}$ (mean $\pm \mathrm{SD}$, $7.9 \pm 0.3$ weeks postoperatively), of whom $60 \%$ did not receive RAI. All patients who received RAI maintained an unstimulated thyroglobulin level of $\leq 0.2$ $\mathrm{ng} / \mathrm{ml}$ at 6 months and at 1 year postoperatively. Of those who did not receive RAI, $96.7 \%$ maintained an unstimulated thyroglobulin level of $\leq 0.2 \mathrm{ng} / \mathrm{ml}$ at 6 months, as compared with $94.1 \%$ at 1 year postoperatively.

Subsequently, early postoperative unstimulated thyroglobulin levels were evaluated after being categorized as $\leq 0.2 \mathrm{ng} / \mathrm{ml}$ (no evidence of disease), $>0.2$ to $2.0 \mathrm{ng} / \mathrm{ml}$ (microscopic benign remnant or disease), or $>2.0 \mathrm{ng} / \mathrm{ml}$ (macroscopic benign remnant or per- sistent disease), and patients were stratified based on whether they received RAI. Overall, 69\% of patients with a postoperative unstimulated thyroglobulin level of $>0.2$ to $2.0 \mathrm{ng} / \mathrm{ml}$ achieved a goal of $\leq 0.2 \mathrm{ng} / \mathrm{ml}$ at 6 months if they received RAl, as compared with only $15.4 \%$ if they didn't receive RAI $(P=0.002)$. Of those with an early postoperative unstimulated thyroglobulin level of $>2.0 \mathrm{ng} / \mathrm{ml}$, only $30.8 \%$ achieved a goal of $\leq 0.2 \mathrm{ng} / \mathrm{ml}$ at 6 months if they received RAI, as compared with only $25.0 \%$ if they did not receive $\mathrm{RAI}(\mathrm{P}=0.4)$.

\section{Conclusions}

In this cohort of papillary thyroid cancer patients, half achieved an unstimulated thyroglobulin level $\leq 0.2 \mathrm{ng} / \mathrm{ml}$ by 8 weeks postoperatively, suggesting adequate initial surgical resection in this group. Of those with evidence of microscopic benign thyroid tissue remnant or persistent disease, administration of RAl led to a higher frequency of no biochemical evidence of disease by 6 months postoperatively. Finally, patients with the highest levels of unstimulated thyroglobulin (i.e. $>2.0 \mathrm{ng} / \mathrm{ml}$ ) rarely achieved cure, regardless of RAl use. These findings suggest that early postoperative unstimulated thyroglobulin levels can be used to predict the utility of RAI in patients with papillary thyroid cancer.

\section{COMMENTARY}

Results from this study indicate that the use of early postoperative unstimulated thyroglobulin levels can be considered a predictor of utility of RAI in patients who underwent surgery for papillary thyroid cancer. Overall, patients whose early unstimulated thyroglobulin levels indicate excellent response from surgery do not require RAl; however, patients with evidence of microscopic disease appear to benefit from RAI treatment. Finally, patients with evidence of macroscopic disease, as evidenced by early unstimu- lated thyroglobulin levels, warrant further investigation to determine the source of persistent disease.

Prior studies have focused mostly on the utility of stimulated thyroglobulin levels postoperatively to guide clinical decision making regarding the administration of RAI in patients with differentiated thyroid cancer. In a study of 129 patients with low- and intermediate-risk papillary thyroid cancer, Orlov et al. (7) recommended that patients with a stimulated 
thyroglobulin level $<1 \mu \mathrm{g} / \mathrm{L}$ should not receive RAl, while patients with a stimulated thyroglobulin level of $>5 \mu \mathrm{g} / \mathrm{L}$ should receive RAI routinely. In addition, they proposed that those with stimulated thyroglobulin levels of 1 to $5 \mu \mathrm{g} / \mathrm{L}$ should be further evaluated on the basis of repeat stimulated thyroglobulin levels, pathologic features, and patients' comorbidities and attitude toward RAI. Similarly, this study by McDow et al. (5) proposes to use a strategy using thyroglobulin levels for clinical management and decision making, but exploiting unstimulated rather than stimulated levels, which is a more practical approach.

The results of this study have significant implications for the clinical management of papillary thyroid cancer patients, as measurement of unstimulated thyroglobulin in the early postoperative period may help in decision making regarding the administration of RAl earlier rather than later. This practice would therefore result in minimizing overtreatment of low-risk thyroid cancer and on the flip side recognize the presence of macroscopic persistent disease early in the surveillance process.
Strengths of this study include availability of serial TSH and thyroglobulin measurements, as well as neck ultrasounds, and details on receipt of RAI. Limitations of the study include its retrospective nature, which cannot take fully into account any selection bias, its relatively short follow-up, and its lack of information on histopathology, such as the presence of aggressive variants. Additionally, as this study was conducted in patients seen at an institution with high-volume endocrine surgeons, results may not be generalizable to lower-volume institutions, as the quality of surgical resection may vary.

Future studies should explore assessment of utility of early postoperative unstimulated and stimulated thyroglobulin levels using population-based data with longer follow-up and consider investigating differences among patients with papillary and follicular thyroid cancer. These efforts will help in developing personalized criteria for risk stratification and selection of treatment with radioactive iodine in patients with papillary thyroid cancer.

\section{References}

1. Haugen BR, Alexander EK, Bible KC, Doherty GM, Mandel SJ, Nikiforov YE, Pacini F, Randolph GW, Sawka AM, Schlumberger M, Schuff KG, Sherman SI, Sosa JA, Steward DL, Tuttle RM, Wartofsky L 2016 2015 American Thyroid Association management guidelines for adult patients with thyroid nodules and differentiated thyroid cancer: the American Thyroid Association guidelines task force on thyroid nodules and differentiated thyroid cancer. Thyroid 26:1-133.
2. Castagna MG, Maino F, Cipri C, Belardini V, Theodoropoulou A, Cevenini G, Pacini F 2011 Delayed risk stratification, to include the response to initial treatment (surgery and radioiodine ablation), has better outcome predictivity in differentiated thyroid cancer patients. Eur J Endocrinol 165:441446.

3. Han JM, Kim WB, Yim JH, Kim WG, Kim TY, Ryu JS, Gong G, Sugn TY, Yoon JH, Hong SJ, et al. 2012 Long-term clinical outcome of differentiated thyroid cancer patients with undetectable stimulated thyroglobulin level one year after initial treatment. Thyroid 22:784-90. 
4. Giovanella L, Maffioli M, Ceriani L, De Palma D, Spriano G 2009 Unstimulated high sensitive thyroglobulin measurement predicts outcome of differentiated thyroid carcinoma. Clin Chem Lab Med 47:1001-1004.

5. McDow AD, Shumway CM, Pitt SC, Schneider DF, Sippel RS, Long KL 2019 Utility of early postoperative unstimulated thyroglobulin in influencing decision making in patients with papillary thyroid carcinoma. Ann Surg Oncol. Epub 2019 Jul 2.
6. Bates MF, Lamas MR, Randle RW, Long KL, Pitt SC, Schneider DF, Sippel RS 2018 Back so soon? Is early recurrence of papillary thyroid cancer really just persistent disease? Surgery 163:118-123.

7. Orlov S, Salari F, Kashat L, Freeman JL, Vescan A, Witterick IJ, Walfish PG 2015 Post-operative stimulated thyroglobulin and neck ultrasound as personalized criteria for risk stratification and radioactive iodine selection in low- and intermediaterisk papillary thyroid cancer. Endocrine 50:130-137. 